Cell Research (2003); 13(5):375-383

http://www.cell-research.com

\title{
Effects of MEK inhibitor U0126 on meiotic progression in mouse oocytes: microtuble organization, asymmetric division and metaphase II arrest
}

\author{
Chao Tong ${ }^{1}$, Heng Yu Fan ${ }^{1}$, Da Yuan $\mathrm{Chen}^{1}$, Xiang Fen Song ${ }^{1}$, Heide Schatten $^{2}$, Qing Yuan Sun ${ }^{1, *}$ \\ 1 State Key Laboratory of Reproductive Biology, Institute of Zoology, Chinese Academy of Sciences, Beijing 100080, China. \\ E-mail:sunqy@panda.ioz.ac.cn \\ 2 Department of Veterinary Pathobiology, University of Missouri-Columbia, MO 65211, USA.
}

\begin{abstract}
In this study we used U0126, a potent and specific inhibitor of MEK, to study the roles of MEK/ERK/p90 ${ }^{r s k}$ signaling pathway in the meiotic cell cycle of mouse oocytes. The phosphorylation of MAP kinase and $\mathrm{p} 90^{r s k}$ in the oocytes treated with $1.5 \mu \mathrm{M} \mathrm{U} 0126$ was the same as that in oocytes cultured in drug-free medium. With $1.5 \mu \mathrm{M}$ U0126 treatment, the spindles appeared normal as they formed in oocytes, but failed to maintain its structure. Instead, the spindle lost one pole or elongated extraordinarily. After further culture, some oocytes extruded gigantic polar bodies $(>30 \mathrm{\mu} \mathrm{m})$ that later divided into two small ones. Some oocytes underwent symmetric division and produced two equal-size daughter cells in which normal spindles formed. In oocytes with different division patterns, MAP kinase was normally phosphorylated. When the concentration of U0126 was increased to $15 \mu \mathrm{M}$, the phosphorylation of both MAPK and $\mathrm{p} 90^{r s k}$ were inhibited, while symmetric division was decreased. When incubating in medium containing $15 \mu \mathrm{M}$ U0126 for $14 \mathrm{~h}$, oocytes were activated, but part of them failed to emit polar bodies. MII oocytes were also activated by $15 \mu \mathrm{M} \mathrm{U} 0126$, at the same time the dephosphorylation of MAP kinase and p90 ${ }^{r s k}$ was observed. Our results indicate that 1) MEK plays important but not indispensable roles in microtubule organization; 2) MEK keeps normal meiotic spindle morphology, targets peripheral spindle positioning and regulates asymmetric division by activating some unknown substrates other than MAP kinase $/ \mathrm{p} 90^{r s k}$; and 3) activation of MEK/ERK/p90 ${ }^{r s k}$ cascade maintains MII arrest in mouse oocytes.
\end{abstract}

Keywords: kinases, signal transduction, oocyte development, fertilization, meiosis.

\section{INTRODUCTION}

The meiotic cell cycle of oocytes is regulated by a cascade of protein phosphorylation/ dephosporylation events. Two isoforms of MAP kinases, ERK1 (p44) and ERK2 (p42), appear to play a pivotal role in this orchestration $[1,2]$. MAP kinases are a family of Ser/Thr protein kinases that are activated by MEK (MAP kinase kinase) phosphorylation of both tyrosine and threonine residues. The activation of MEK is regulated by MOS, a germ cell-specific Ser/Thr protein kinase. Among many substrates for MAP kinases, $\mathrm{p} 90^{\text {rsk }}$ was the first to be discovered[3]. In Xenopus oocytes, the main effects of MAP kinase, including S phase suppression, MII arrest

\footnotetext{
*Correspondence: Dr. Qing Yuan SUN, State Key Laboratory of Reproductive Biology, Institute of Zoology, Chinese Academy of Sciences, Beijing 100080, China, Tel: 8610-6256-3923; Fax: 86106256-5689; E-mail: sunqy@panda.ioz.ac.cn

Received Dec-12-2002 Revised Aug-21-2003 Accepted Sep-25-2003
}

maintenance, and spindle formation regulation, are mediated by $\mathrm{p} 90^{r s k}[4,5]$.

There is an evidence suggesting that MAPK pathway is involved in the regulation of microtubule organization during the meiosis of oocytes. Mouse oocytes injected with antibody against MOS failed to assemble a meiotic spindle[6]. In maturing $\operatorname{mos}^{-/}$oocyte MAP kinase could not be activated throughout meiosis, which resulted in the shape alteration of spindle[7,8]. In mouse oocytes, MAP kinase is specifically associated with the microtubule-organizing centers (MTOCs) present at the spindle poles and in the cytoplasm[9], which suggests that MAP kinase might involve in controlling spindle assembly and microtubular configurations. Reports in other species also suggested the important roles of MAP kinase in microtubule organization[10]. Our previous study [11] showed that MAP kinase might be involved in microtubule nucleation by $\gamma$-tubulin in pig oocytes. However, 
it was reported that mouse oocytes deficient in MAP kinase activity could still divide and release polar bodies [6,12-14]. Until now, the targets of MAP kinase in the meiotic spindle remain unclear. Therefore, the roles of MAP kinase in spindle organization is still a question to be elucidated.

Oocyte meiosis is characterized by asymmetric division. But the first meiotic division is symmetric in some mos $^{-/}$oocytes[8]. In wild-type oocytes the spindle formed in the center of oocytes and migrated to the cortex before meiotic division. But in mos $^{-/-}$oocytes that underwent asymmetric division, the centrally formed spindle failed to migrate, instead, it elongated in place[14]. Although many reports show that MOS participates in oocyte asymmetric division, the downstream molecule that mediates this event is still unclear.

The metaphase II arrest is another characteristic of meiosis in almost all mammalian oocytes. It is well established that the MII arrest is maintained by cytostatic factor (CSF) activity, with MOS/MEK/ERK cascade being its essential components[4,10,12,13,15]. Although existing evidence has suggested that MAP kinase is important for metaphase arrest, almost all data come from $\operatorname{mos}^{-/}$oocytes or other models indication MAP kinase is not activated during the entire maturation process. It is still unknown whether the MII oocytes with activated MAP kinase could be released from the arrest when MEK is specifically inhibited.

U0126, a potent and specific inhibitor of MEK1 and MEK2, has little effect on other kinases such as Cdk2, ERK, JNK, MEKK and Raf[16]. In porcine oocytes, the inhibition of ERK activity with U0126 during the meiosis I/meiosis II transition suppressed chromosome separation, first polar body emission and formation of the metaphase II spindle[17]. When incubated in a medium with U0126, part of porcine oocytes completed the second meiotic division and entered into interphase[18]. In cumulus-enclosed mouse oocytes, U0126 produced a dose-dependent inhibitory effect on FSH-induced meiotic resumption[19].

This report shows the novel effects of U1026 on meiotic cell cycle progression in mouse oocytes, including ERK and $\mathrm{p} 90^{r s k}$ activation, disturbance of microtubule organization, PB extrusion abnormality, and MII arrest. The findings provide further understanding of the roles of MAP kinase signaling pathway in the regulation of meiotic cell cycle progression in mammalian oocytes.

\section{MATERIALS AND METHODS}

\section{Oocyte collection and culture}

Fully grown germinal vesicle-intact oocytes were collected from ovaries of 4-6 week-old Kunming mice $48 \mathrm{~h}$ after the females were injected with $10 \mathrm{IU}$ pregnant mare' s serum gonadotropin (PMSG). Cumulus-free and GV-intact oocytes were released from antral follicles by puncturing the follicles with a needle in M2 medium (Sigma) supplemented with $60 \mathrm{mg} / \mathrm{ml}$ penicillin and $50 \mu \mathrm{g} / \mathrm{ml}$ streptomycin. All cultures were carried out in $\mathrm{M} 2$ medium at $37^{\circ} \mathrm{C}$ in a humidified atmosphere of $5 \% \mathrm{CO}_{2}$.

Metaphase II-arrested eggs were obtained from mice of the same strain. Females were superovulated by intraperitoneal injection of 10 IU of PMSG, and $48 \mathrm{~h}$ later, they were injected with $10 \mathrm{IU}$ of human chorionic gonadotrophin (hCG). Mice were sacrificed and oviducts were removed at $15 \mathrm{~h}$ post-hCG injection. Using a pair of fine forceps to tear the oviducts, cumulus masses were collected in M2 medium. The cumulus cells surrounding the eggs were removed by a brief exposure to $300 \mathrm{IU} / \mathrm{ml}$ hyaluronidase and repeated pipetting, followed by three washes in M2 medium.

\section{Parthenogenetic activation}

Parthenogenetic activation of mouse oocytes was performed as we previously reported[20]. The denuded eggs were treated with $5 \mu \mathrm{M}$ A23187 for $5 \mathrm{~min}$, and then incubated in M2 medium containing $10 \mu \mathrm{g} / \mathrm{ml} \mathrm{CHX}$. After 2, 4 and $8 \mathrm{~h}$ of culture, treated eggs were examined with a phase contrast microscope. The emission of the second polar body and the formation of pronucleus were examined.

\section{Confocal microscopy}

After removal of zona pellucida (ZP) in acidified Tyrode's solution ( $\mathrm{pH} 2.5$ ), eggs were fixed in $3 \%$ formaldehyde, $2 \%$ sucrose in PBS for $30 \mathrm{~min}$ and then incubated in incubation buffer $(0.5 \%$ Triton X-100 in $20 \mu \mathrm{M}$ Hepes, pH 7.4, $3 \mu \mathrm{M} \mathrm{MgCl}_{2}, 50 \mu \mathrm{M} \mathrm{NaCl}, 300 \mu \mathrm{M}$ sucrose, $0.02 \% \mathrm{NaN} 3$ ) for $30 \mathrm{~min}$. After being treated in methanol for $5 \mathrm{~min}$ at $-20{ }^{\circ} \mathrm{C}$, the eggs were washed in washing buffer (PBS with 0 . $1 \%$ Tween 20 and $0.01 \%$ Triton X-100) for three times and then incubated with 1: 50 diluted FITC-conjugated mouse anti-a tubulin antibody (Sigma) for $1 \mathrm{~h}$. The eggs were rinsed three times and incubated with $10 \mathrm{mg} / \mathrm{ml}$ propidium iodide. Finally, the eggs were mounted on glass slides and examined using a TCS-4D laser scanning confocal microscope (Leica Microsystems).

\section{Western blot analysis}

Eggs were collected in SDS sample buffer after treatment with different stimuli at different times, heated to $100{ }^{\circ} \mathrm{C}$ for $4.5 \mathrm{~min}$, and frozen at $-20^{\circ} \mathrm{C}$ until use. Each sample contained 30 eggs. The total proteins were separated by SDS-PAGE with a $4 \%$ stacking gel and $10 \%$ separating gel at $90 \mathrm{~V}, 0.5 \mathrm{~h}$ and $120 \mathrm{~V}, 2 \mathrm{~h}$ respectively and electrophoretically transferred to nitrocellulose membrane (Gelmen, poresize $0.45 \mu \mathrm{m}$ ) for $2 \mathrm{~h}, 200 \mathrm{~mA}$, at $4 \mathrm{oC}$. Following transfer, washing in TBS (10 $\mu \mathrm{M}$ Tris, $150 \mu \mathrm{M} \mathrm{NaCl}, \mathrm{pH}$ 7.4) twice and blocking in 5\% skimmed milk in TBST (TBS containing $0.1 \%$ Tween20 ) at $4^{\circ} \mathrm{C}$ overnight, the memberane was incubated in TBST containing 1:1000 polyclonal anti-phospho-ERK antibody (Santa Cruz 
Biotechnology, Santa Cruz, CA) at $37^{\circ} \mathrm{C}$ for $2 \mathrm{~h}$. After washing three times in TBST, 10 min each, the membrane then was incubated with HRP-conjugated goat-anti-mouse IgG (Jackson Immunoresearch Laboratories, INC, PA) diluted 1:1000 in TBST at $37^{\circ} \mathrm{C}$ for $1 \mathrm{~h}$. The membrane was washed three times in TBST, $10 \mathrm{~min}$ each, and was processed by using the ECL detection system.

For reprobing, the membrane was washed in stripping buffer (100 $\mu \mathrm{M}$ b-mercatoethanol, 20\% SDS, 62.5 $\mu \mathrm{M}$ Tris pH6.7) at $50^{\circ} \mathrm{C}$ for 30 min to strip off bound antibody after ECL detection. The membrane was reprobed with polyclonal rabbit anti-ERK2 antibody (Santa Cruz Biotechnology, Santa Cruz, CA) diluted 1:300 and polyclonal rabbit anti-p90rsk antibody (Santa Cruz Biotechnology, Santa Cruz, CA) diluted 1:500, using the same procedure as described above. All experiments were repeated at least three times.

\section{Statistical analysis}

All percentages from 2 or 3 repeated experiments were expressed as mean \pm SEM and the number of oocytes observed was labeled in brackets as $(\mathrm{n}=)$. The rates of $\mathrm{PB} 1$ emission and symmetric division were subjected to arcsin transformation. The transformed data were analyzed by ANOVA followed by Student-Newman-Keuls test. Differences at $p<0.05$ were considered to be statistically significant.

\section{RESULTS}

Division patterns and activity of MAP kinase and p90 $0^{\text {rsk }}$ in oocytes treated with U0126

In order to study the effects of MEK/ERK/p90 ${ }^{\text {rsk }}$ cascade inhibition on meiotic maturation of mouse oocytes, cumulus-free and GV intact oocytes were incubated in M2 medium containing 1.5 $\mu$ M U0126[19]. The culture medium containing U0126 was renewed every $12 \mathrm{~h}$ to prevent the possible degradation of U0126. Some oocytes were cultured in drug-free medium as control. As shown in Fig 1A, there was no significant difference in PB1 emission rate between oocytes cultured with or without U0126 $14 \mathrm{~h}$ after maturation culture $(74.9 \pm 2.4 \%(\mathrm{n}=307)$ in U0126 treated groups, and $75.7 \pm 0.44 \%(n=162)$ in drug-free groups. Although part of treated oocytes underwent asymmetric division as in control oocytes, $8.4 \pm 0.32 \%$ oocytes $(n=307)$ underwent symmetric division, resulting in the formation of 2cell-like oocytes, and $36.2 \pm 1.4 \%(n=307)$ extruded extraordinarily big polar bodies $(>30 \mu \mathrm{m})$ that did not undergo degeneration but divided in the following $10 \mathrm{~h}$. To detect the changes of the activity of ERK and $\mathrm{p} 90^{r s k}$, the oocytes at different developmental stages were collected for Western blot analysis. ERK activity and the full phosphorylation of $\mathrm{p} 90^{\text {rsk }}$ could be detected in oocytes after 4 $\mathrm{h}$ incubation with U0126 (Fig 2), the same as in oocytes
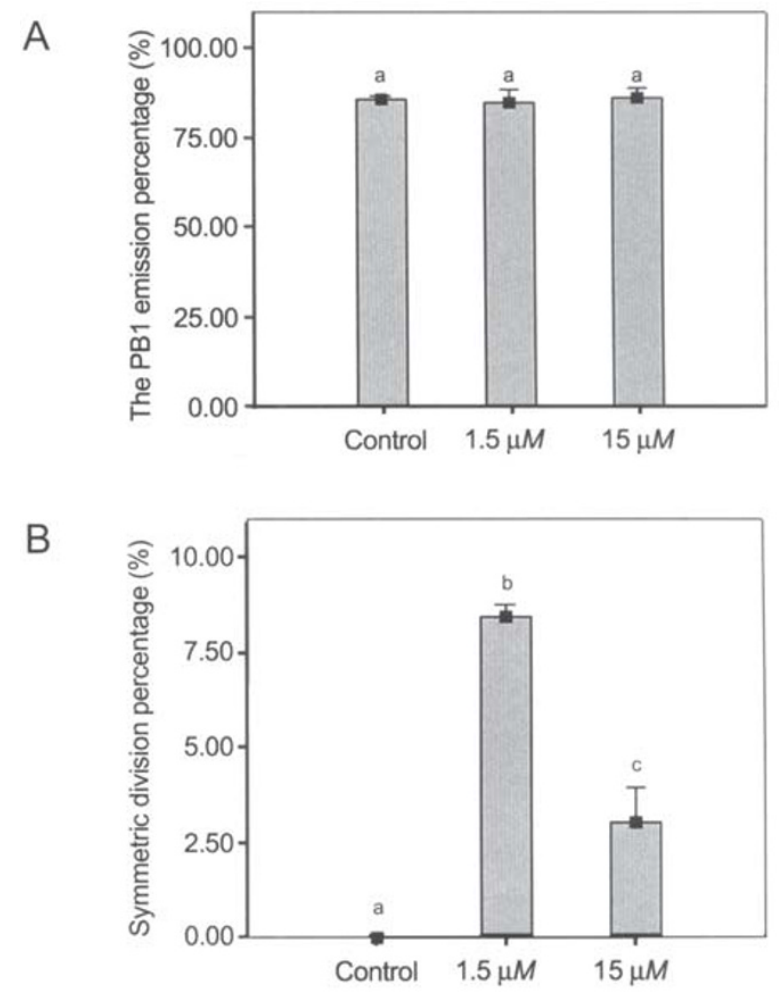

Fig 1. Effect of U0126 on polar body emission and symmetric division of mouse oocytes. Cumulus-free GV oocytes were treated with different concentrations of U0126 (1.5 and $15 \mu \mathrm{M})$ for $14 \mathrm{~h}$. As control, oocytes at GV stage were cultured in a drug-free medium for $14 \mathrm{~h}$. (A) The PB1 emission rates in mouse oocytes treated with U0126. (B) The symmetric division rates in mouse oocytes treated with U0126. The rate was shown as percentage (mean+SEM). Different superscripts $(\mathrm{a}, \mathrm{b}$, and $\mathrm{c}$ ) denote statistical difference at a $\mathrm{P}$ $<0.05$ level of significance.

cultured in a drug-free medium (Fig 3). After $8 \mathrm{~h}$ incubation, ERK of the oocytes was completely activated and its activation maintained until $24 \mathrm{~h}$ after incubation in both $1.5 \mu \mathrm{M}$ U0126 treated and drug-free medium.To detect whether the oocytes with unusual division patterns were more sensitive to U0126, the oocytes with different division patterns (2-cell like, 2-PB like, normalMI like, no-PB) were collected at $15 \mathrm{~h}$ is incubation with $1.5 \mu \mathrm{M}$ U0126 and the ERK activity was analyzed. Just as in the control, high ERK phosphorylation was detected in each sample (Fig 4).

Since $1.5 \mu \mathrm{M}$ U0126 does not inhibit the MEK/ ERK/p90rsk cascade, higher concentrations of U0126 were used and PB1 emission was observed. We found that there was no significant difference in PB1 emission rates between the oocytes treated with 1.5 and $15 \mu \mathrm{M}$ U0126 [74.9 $\pm 2.4 \%(n=307)$ and $76.0 \pm 1.8 \%(n=200)$, respectively](Fig 1A), However, the rate of symmetric division in oocytes treated with $15 \mu \mathrm{M} \mathrm{U} 0126$ (3.02 \pm 


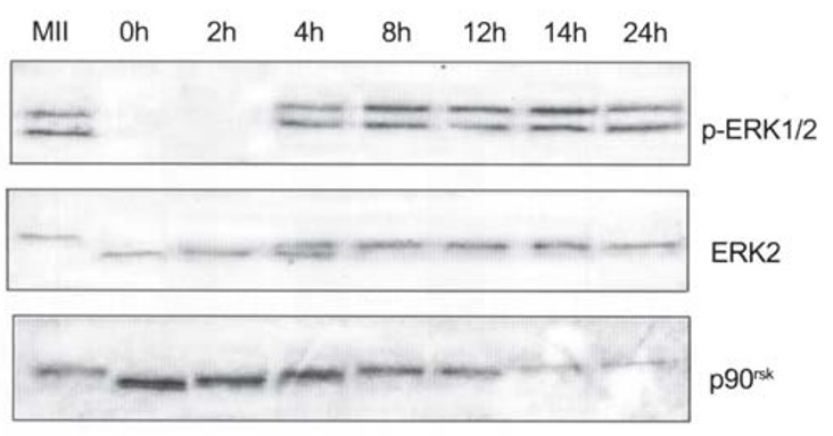

Fig 2. Effect of $1.5 \mu \mathrm{M}$ U0126 on MAP kinase and p90rsk phosphorylation in mouse oocytes. Samples for immunoblotting were collected after the GV-intact oocytes were treated with $1.5 \mu$ M U0126 for $0,2,4,8,12,14$, and $24 \mathrm{~h}$. MII oocytes were also collected as a positive control. MAP kinase was activated $4 \mathrm{~h}$ after maturation. A basic phosphorylation of $\mathrm{p} 90^{r s k}$ was observed in $\mathrm{GV}$ oocytes and the full phosphorylation was detected $4 \mathrm{~h}$ after culture. Each lane represent the protein extracted from 30 cells.

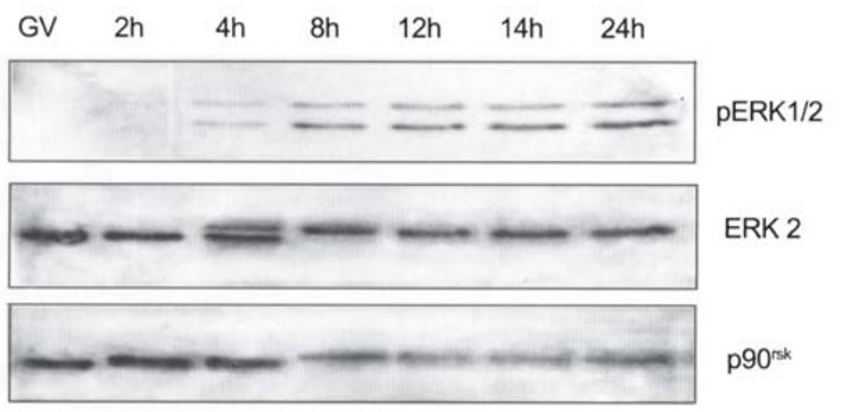

Fig 3. MAP kinase and $\mathrm{p} 90^{r s k}$ phosphorylation during mouse oocytes maturation. Samples for immunoblotting were collected after the GV-intact oocytes were cultured for $0,2,4,8,12,14$, and $24 \mathrm{~h}$. MAP kinase was activated $4 \mathrm{~h}$ after maturation. A basic phosphorylation of $\mathrm{p} 90^{r s k}$ was observed in GV oocytes and the full phosphorylation was detected $8 \mathrm{~h}$ after culture.

$90 \%(\mathrm{n}=200))$ was significantly lower than that in those oocytes treated with $1.5 \mu$ M U0126 (Fig 1B). Western blot analysis showed that the activity of MAP kinase and migration of $\mathrm{p} 90^{r s k}$ could not be detected within $24 \mathrm{~h}$ after incubation with $15 \mu \mathrm{M}$ U0126 (Fig 5). Therefore, $15 \mu$ $\mathrm{M}$ is the effective concentration for U0126 to inhibit MAP kinase and $\mathrm{p} 90^{\text {rsk }}$ activation.

To examine whether both daughter cells of the 2cell-like oocytes were able to undergo the second meiotic division, the oocytes were treated with A23187+CHX. Both cells divided $2 \mathrm{~h}$ after activation and no obvious asymmetric division was observed. (Fig 6)

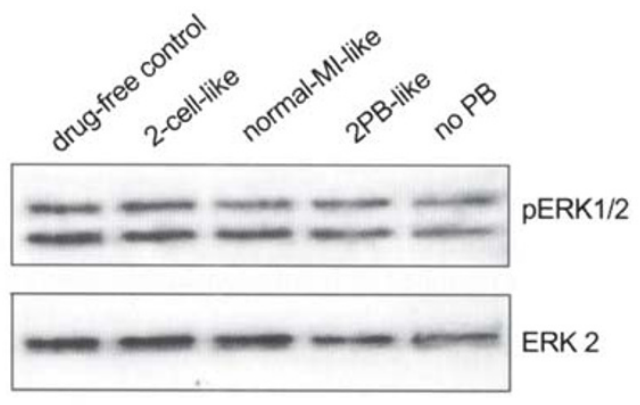

Fig 4. MAP kinase activity in oocytes with different division patterns. Cumulus-free GV oocytes were cultured in medium containing $1.5 \mu \mathrm{M} \mathrm{U} 0126$ for $15 \mathrm{~h}$. The oocytes with different division patterns (2-cell like, 2-PB like, normal-MI like, no-PB) were collected respectively. GV oocytes cultured in a drug-free medium for $14 \mathrm{~h}$ and then collected as control group. MAP kinase phosphorylation in the oocytes with different division patterns. MAP kinase was fully phosphorylated in the oocytes with different division patterns and drug-free control.

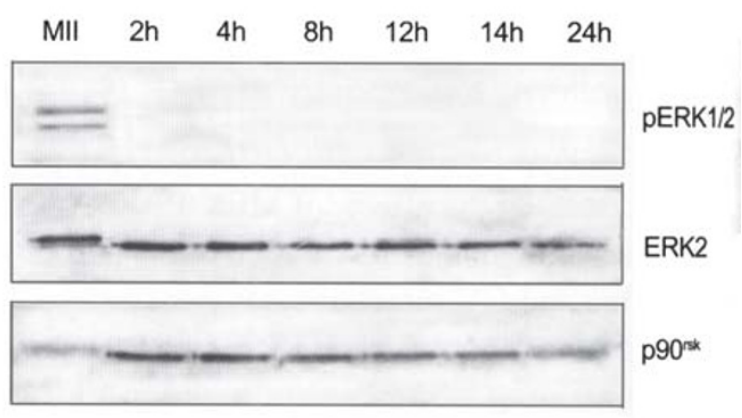

Fig 5. Effect of $15 \mu \mathrm{M}$ U0126 on MAP kinase and p90 $0^{r s k}$ phosphorylation in mouse oocytes. Samples for immunoblotting were collected after the GV intact oocytes were treated with $15 \mu \mathrm{M} \mathrm{U} 0126$ for $0,2,4,8,12,14$, and $24 \mathrm{~h}$. MII oocytes were also collected as a positive control. $15 \mu \mathrm{M} \mathrm{U} 0126$ inhibited MAP kinase activation and full phosphorylation of $\mathrm{p} 90^{r s k}$.

\section{Microtubule Dynamics in Oocytes Treated with U0126}

Since MEK/ERK/p90 ${ }^{r s k}$ cascade affects the division pattern of the oocytes, microtubule organization was examined in oocytes treated with 1.5 or $15 \mu M \mathrm{U} 0126$. In oocytes treated with $1.5 \mu M$ U0126, spindles formed normally as in drug-free control $8 \mathrm{~h}$ after incubation (Fig 7A). However, when the incubation time was prolonged, the intracellular position or morphology of the spindle changed. Three phenomena existed: 1) the spindle elongated and one pole closest to the cortex moved while 2- 

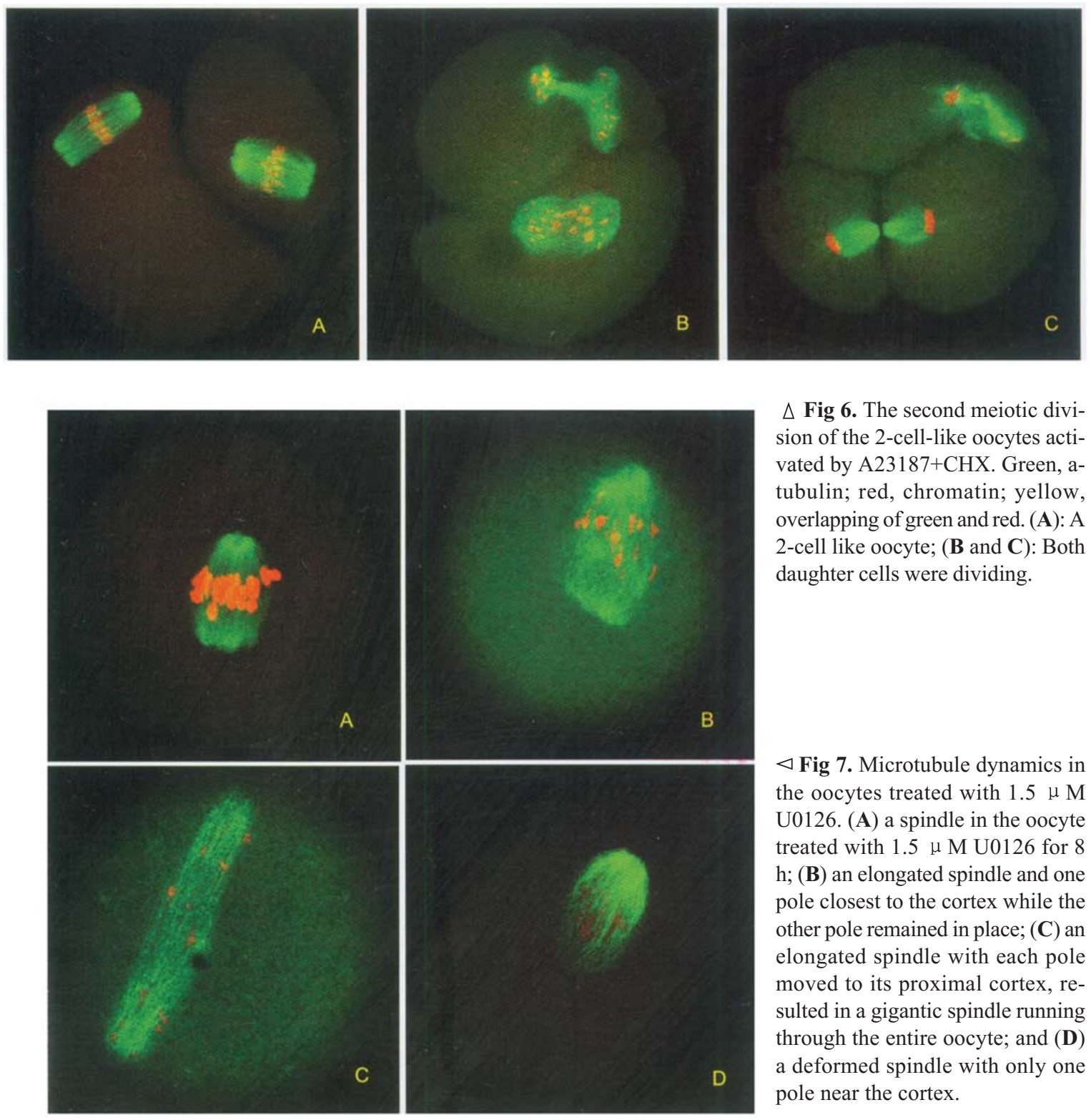

$\Delta$ Fig 6. The second meiotic division of the 2-cell-like oocytes activated by A23187+CHX. Green, atubulin; red, chromatin; yellow, overlapping of green and red. (A): A 2-cell like oocyte; (B and $\mathbf{C})$ : Both daughter cells were dividing.

$\triangleleft$ Fig 7. Microtubule dynamics in the oocytes treated with $1.5 \mu \mathrm{M}$ U0126. (A) a spindle in the oocyte treated with $1.5 \mu \mathrm{M} \mathrm{U} 0126$ for 8 $\mathrm{h}$; (B) an elongated spindle and one pole closest to the cortex while the other pole remained in place; $(\mathbf{C})$ an elongated spindle with each pole moved to its proximal cortex, resulted in a gigantic spindle running through the entire oocyte; and (D) a deformed spindle with only one pole near the cortex.
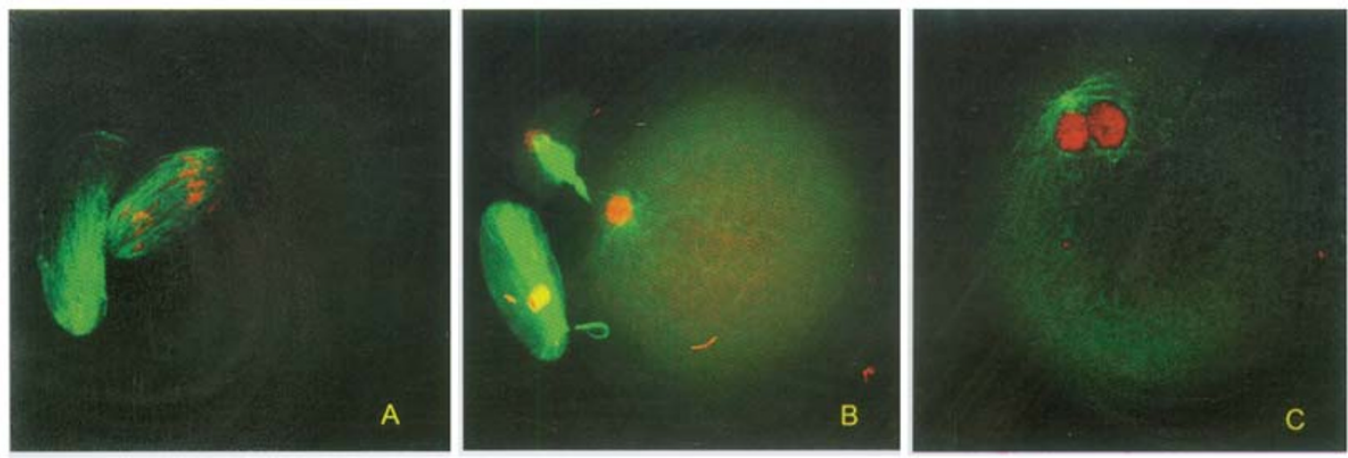

Fig 8. The GV oocytes incubated in a medium containing $15 \mu$ M U0126. (A and B) the oocytes were activated after $14 \mathrm{~h}$ incubation; and $(\mathbf{C})$ an oocyte with two pronuclei. 
cell-like oocytes or oocytes with abnormally big polar bodies were formed. The oocytes arrested at MII stage, with normal the another remained in place (Fig 7B); 2) the spindle elongated but each pole moved to its proximal cortex, resulting in a gigantic spindle running through the oocyte(Fig 7C); and 3) the spindle was deformed with only one pole near the cortex (Fig 7D). Microtubule dynamice were the same in oocytes treated with 1.5 or
$15 \mu \mathrm{M} \mathrm{U} 0126$ before $12 \mathrm{~h}$ incubation (data not shown). However, a large proportion of the oocytes [68.9 \pm 1 . $45 \%(n=153)]$ incubated with $15 \mu$ M U0126 did not arrest at MII stage, but entered interphase with formation of pronuclei and diffusion of microtubules (Fig 8A-B). The PB2 emission was blocked in some activated oocytes $[11.0 \pm 0.15 \%(n=91)]$. As a result, two pronuclei were observed in these oocytes (Fig 8C).
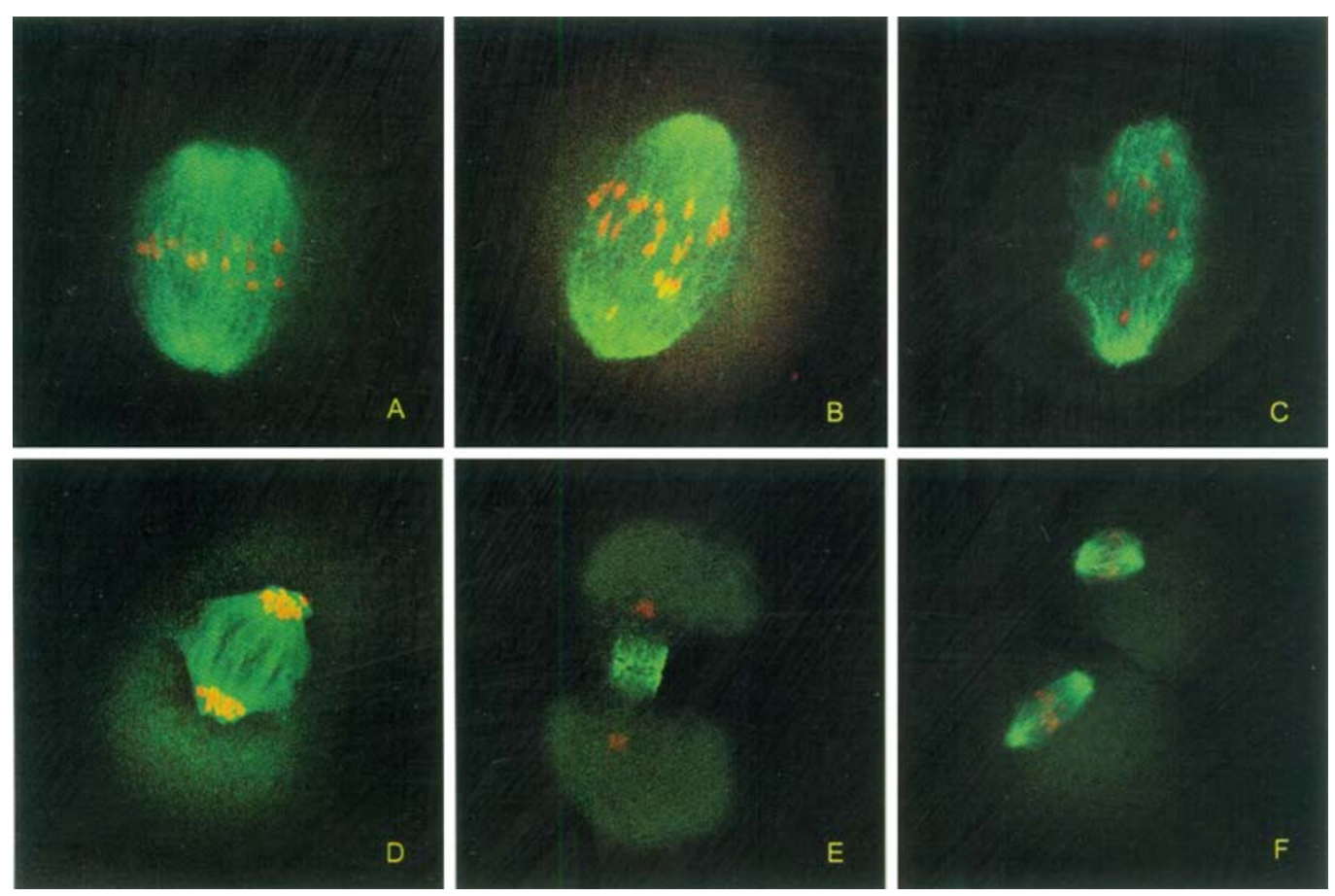

Fig 9. Microtubule dynamics in the oocytes with symmetric division. (A, B and C) spindles elongated extraordinarily; (D and E) oocytes with symmetric division; and (F) each daughter cell formed a normal spindle.

Microtubule dynamics were also traced in the oocytes with equal division. After $8 \mathrm{~h}$ incubation in $1.5 \mu$ M U0126, the spindle formed displaying normal morphology (Fig 7A). It elongated during metaphase I /anaphase I transition rather than migrated to the cortex as observed in normal oocytes (Fig 9A-C). As a result, spindles in both daughter cells (Fig 9D-F).

\section{MAP kinase and $p 90^{r s k}$ activity in MII eggs treated with U0126}

To examine the effect of MEK inhibition on MII arrest and MAP kinase/p90 $0^{r s k}$ phosphorylation, MII eggs

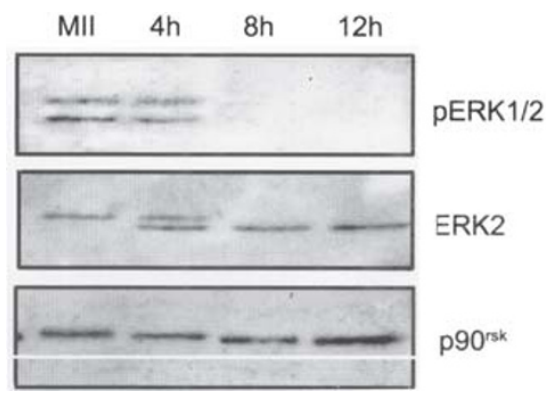

Fig 10. Effect of $15 \mu \mathrm{M} \mathrm{U} 0126$ on MAP kinase and p90 $0^{\text {rsk }}$ phosphorylation in mouse oocytes at MII stage. Samples for immunoblotting were collected after the MII oocytes were treated with $15 \mu \mathrm{M} \mathrm{U} 0126$ for $0,4,8$, and $12 \mathrm{~h}$, respectively. MAP kinase and $\mathrm{p} 90^{r s k}$ were dephosphorylated after $4 \mathrm{~h}$ treatment. 
were treated with $15 \mu \mathrm{M} \mathrm{U} 0126$ and collected every 4 $\mathrm{h}$ for nuclear status examination and for Western blot analysis. After $12 \mathrm{~h}$ of incubation, pronuclei were formed in $38.8 \pm 7.8 \%(\mathrm{n}=131)$ of the treated eggs. The results of Western blot showed that MAP kinase activity was decreased and the $\mathrm{p} 90^{r s k}$ was gradually dephosphorylated (Fig 10).

\section{DISCUSSION}

In both vertebrates and invertebrates, meiotic divisions of oocytes are typically asymmetric. The size difference between two daughter cells is typically a consequence of asymmetric positioning of the spindle before cytokinesis. In mouse oocytes, the meiotic spindle forms in the center of the cell, which does not elongate during anaphase but migrates to the cortex just before polar body extrusion[21]. In mos ${ }^{-/}$mouse oocytes, the spindle shape is altered and the spindle fails to translocate to the cortex. As a result, in these oocytes the first meiotic division resembles mitotic cleavage and produces two cells of the same size[22]. Inhibition of protein synthesis frequently results in a similar phenotype[23] in starfish oocytes. In the present study, when the oocytes were treated with $1.5 \mu \mathrm{M} \mathrm{U} 0126$, abnormal division was similar to that observed in mos $^{-/-}$oocytes. However, in oocytes treated with $1.5 \mu \mathrm{M} \mathrm{U} 0126$, even in those with typical abnormal division patterns, MAP kinase was still activated as in control oocytes. Furthermore, although MAP kinase could be inhibited by a high concentration of U0126, the rate of symmetric division did not increase. It is apparent that MAP kinase activity does not insure the asymmetric division in oocytes. Interestingly, the $\operatorname{mos}^{-/-}$oocytes have the same phenotype as oocytes treated with U0126 shown here. Since MEK is a downstream molecule of MOS and it is the only target of U0126, it is clear that the depletion of MEK activity, rather than MAP kinase activity, is responsible for the abnormality in meiotic division. It is possible that MEK is involved in the asymmetric cytokinesis of oocytes through substrate of unknown nature and MEK could activate it by the domains different from those responsible for MAP kinase activation. Different concentrations of U0126 may exert diverse effects on MEK configuration and make MEK activate one of its substrates, but not the others. This may be the possible reason of why the incidence of symmetrical division was decreased after the treatment of U0126 with high concentration.

In animal cells, the position of spindle determines the plane of cleavage[24,25]. In our experiments, some spindles in U0126-treated oocytes elongated extraordinarily at metaphase I/ anaphase I transition rather than translocating to the cortex as seen in normal oocytes. As a result, symmetric division took place. During mitosis, spindle positioning involves a cortical-microtubule capture mechanism and several proteins are involved in the interaction between microtubules and the cortex[26,27]. In Saccharomyces cerevisiae, microtubule cortical attachment includes mediation by actin, Kar9p, Bud6p and Bnilp[28,29]. Verlhac et al[14] found that actin distribution was disturbed in mos $^{-/-}$oocytes, which lends strong support to our data. The presence of an actin-rich plaque covering the meiotic spindle appears to be a common feature of animal cortices[30-32]. In wild-type oocytes, the actin-rich cortical domain appears progressively, concomitant with spindle migration[33,34]. In mos $^{-/}$oocytes [14], signs of cortical polarization are present only at anaphase and no obvious actin-rich region is found. MEK and its downstream molecules may act on these proteins and regulate the spindle positioning. Further work is necessary to elucidate these interesting results.

We have shown that the MII eggs were released from MII arrest and MAP kinase and p90rsk were dephosphorylated after MEK had been inhibited, suggesting that MEK activity is necessary for maintaining the activity of MAP kinase and p90rsk as well MII arrest. Since MAP kinase dephosphorylation is protein phosphatase dependent, it is possible that there is an equilibrium between MEK and MAP kinase-specific protein phosphatase in the meiotic cell cycle: if MEK activity overcomes protein phosphatase activity, MAP kinase is phosphorylated; alternatively, MAP kinase is dephosphorylated. Verlhac et al[35] showed that MOS activated MAP kinase through two opposite pathways: activation of MEK and inhibition of protein phosphatase. We and others found that inhibition of protein phosphatase by okadaic acid (OA) induced precocious MAP kinase activation in mouse and pig oocytes [36,37]. OA treatment of fertilized mouse or pig eggs resulted in prompt phosphorylation of MAP kinase, disassembly of microtubules around the pronuclear area, chromatin condensation, and nuclear membrane breakdown[38,39]. Although very little is known about the physiological MAPkinase-specific protein phosphatase, the work in Xenopus oocytes shows that a tyrosine phosphatase and a PP2A-like threonine phosphatase mediate MAP kinase inactivation[40]. At MII arrest, both MEK activity and 
the MAP kinase-specific protein phosphatase activity may exist, but the MEK activity is in dominance. When treated with U0126, MEK activity was inhibited and thus MAP kinase was dephosphorylated, causing release of MII arrest.

The data in Xenopus oocytes suggested that the activity of MAP kinase is essential for maintaining the activity of $\mathrm{p} 90^{r s k}[41,42]$. Previous work by us and others also showed that in mouse and rat oocytes, the change of MAP kinase activity is always followed by the change of $\mathrm{p} 90^{r s k}$ activity during meiotic maturation, fertilization, and parthenogenetic activation[43-45]. However, there is no direct evidence that the inhibition of MAP kinase activity at MII arrest leads to the dephosphorylation of p9 $90^{r s k}$. In our experiments, when MAP kinase activity was inhibited by U0126, during the interphase transition of oocytes, dephosphorylation of $\mathrm{p} 90^{r s k}$ was detected by Western blotting, which supports the previous suggestion that $\mathrm{p} 90^{r s k}$ is one of the direct targets of MAP kinase and a key mediator of CSF in oocytes.

In conclusion, the meiotic abnormalities caused by U0126, a specific inhibitor of MEK, imply important regulatory roles of MEK in asymmetric division and the release of the MII arrest induced by U0126 implies an important role of the MEK/ERK/p90 ${ }^{r s k}$ cascade in MII arrest of mouse oocytes.

\section{ACKNOWLEDGEMENTS}

This study was supported by grants from the Special Funds for Major State Basic Research ("973") Project (G1999055902) of China, National Natural Science Foundation of China $(30225010,30170358)$ and Knowledge Innovation Program (KSCX2-SW-303, KSCX-IOZ07) of Chinese Academy of Sciences.

\section{REFERENCES}

1 Fan HY, Tong C, Chen DY, Sun QY. Roles of MAP kinase signaling pathway in oocyte meiosis. Chin Sci Bul 2002; 47:1-7.

2 Abrieu A, Doree M, Fisher D. The interplay between cyclin-B$\mathrm{Cdc} 2$ kinase (MPF) and MAP kinase during maturation of oocytes. J Cell Sci 2001; 114:257-67.

3 Sturgill TW, Ray LB, Erikson E, Maller JL. Insulin-stimulated MAP-2 kinase phosphorylates and activates ribosome S6 kinase II. Nature 1988; 334:715-8.

4 Bhatt RR, Ferrell JJE. The protein kinase p90 RSK is an essential mediator of cytostatic factor activity. Science 1999; 286:1362-7.

5 Gross SD, Schwab MS, Lewellyn AL, Maller JL. Induction of metaphase arrest in cleaving Xenopus embryos by the protein kinase p90rsk. Science 1999; 286:1365-7.
6 Zhao X, Singh B, Batten BE. The role of c-mos proto-oncoprotein in mammalian meiotic maturation. Oncogene 1991; 6:43-9.

7 Araki K, Naito K, Haraguchi S, et al. Meiotic abnormalities of c-mos knockout mouse oocytes: activation after first meiosis or entrance into third meiotic metaphase. Biol Reprod 1996; 55:131524.

8 Choi T, Fukasawa K, Zhou RP, et al. The Mos/ mitogen-activated protein kinases (MAPK) pathway regulates the size and degradation of the first polar body in maturing mouse oocytes. Proc Natl Acad Sci USA 1996; 93:7032-5.

9 Verlhac MH, Pennart HD, Maro B, Cobb MH, Clarke HJ. MAP kinase becomes stably associated at metaphase and is associated with microtubule-organizing centers during meiotic maturation of mouse oocytes. Dev Biol 1993; 158:330-40 .

10 Gordo AC, He CL, Smith S, Fissore RA. Mitogen-activated protein kinase plays a significant role in metaphase II arrest, spindle morphology, and maintenance of maturation promoting factor activity in bovine oocyte. Mol Reprod Dev 2001; 59:106-14 .

11 Sun QY, Lai L, Wu GM, et al. Microtubule assembly after treatment of pig oocyte with taxol: correlation with chromosomes, g-tubulin and MAP kinase. Mol Reprod Dev 2001; 99:1-9.

12 Colledge WH, Carlton MBL, Udy GB, Evans MJ. Disruption of c-mos causes parthenogenetic development of unfertilized mouse eggs. Nature 1994; 370:65-7.

13 Hashimoto N, Watanabe N, Furuta Y, et al. Parthenogenetic activation of oocytes in c-mos-deficient mice. Nature 1994; 370: 68-71.

14 Verlhac MH, Lefebvre C, Guillaud P, Rassinier P, Maro B. Asymmetric division in mouse oocytes: with or without Mos. Curr Biol 2000; 10:1303-6.

15 Svoboda P, Stein P, Hayashi H, Schultz RM. Selective reduction of dormant maternal mRNAs in mouse oocytes by RNA interference. Development 2000; 127:4147-56.

16 Favata MF, Horiuchi KY, Manos E J, et al. Identification of a novel inhibitor of mitogen-activated protein kinase kinase. J Biol Chem 1998; 273:18623-32.

17 Lee J, Miyano T, Moor RM. Localization of phosphorylated MAP kinase during the transition from meiosis I to meiosis II in pig oocytes. Zygote 2000; 8:119-25.

18 Tatemoto H, Muto N. Mitogen-activated protein kinase regulates normal transition from metaphase to interphase following parthenogenetic activation in porcine oocytes. Zygote 2001; 9:15-23.

19 Su YQ, Rubinstein S, Luria A, Lax Y, Breitbart H. Involvement of MEK-mitogen-activated protein kinase pathway in follicle-stimulating hormone-induced but not spontaneous meiotic resumption of mouse oocytes. Biol Reprod 2001; 65:358-65.

20 Tong C, Fan H Y, Chen DY, Sun QY. Parthenogenetic activation of mouse eggs induced by cycloheximide is calcium-dependent and can be blocked by okadaic acid. Acta Zool Sin 2002; 48:749753.

21 Verlhac MH, Kubiak JZ, Clarke HJ, Maro B. Microtubule and chromatin behavior follow MAP kinase activity but not MPF activity during meiosis in mouse oocytes. Development 1994; 120: $1017-25$

22 Choi T, Rulong S, Resau J, et al. Mos/ mitogen-activated protein kinase can induce early meiotic phenotypes in the absence of 
maturation-promoting factor: a novel system for analyzing spindle formation during meiosis I. Proc Natl Acad Sci USA 1996; 93: 4730-5.

23 Galas S, Barakat H, Doree M, Picard A. A nuclear factor required for specific translation of cyclin B may control the accumulation of cyclin B and the timing of first meiotic cleavage in starfish oocytes. Mol Biol Cell 1993; 4:1295-306.

24 Hyman AA. Centrosome movement in the early divisions of Caenorhabditis elegans: a cortical site determining centrosome position. J Cell Biol 1989; 109:1185-93.

25 Rhyu MS, Knoblich JA. Spindle orientation and asymmetric cell fate. Cell 1995; 82:523-6.

26 Lee L, Tirnauer JS, Li JJ, Schuyler SC, Liu JY, Pellman D. Positioning of the mitotic spindle by a cortical- microtubule capture mechanism. Science 2000; 287:2260-2.

27 Sardet C, Prodon F, Dumollard R, Chang P, Chê nevert J. Structure and function of the egg cortex from oogenesis through fertilization. Dev Biol 2002; 241:1-23.

28 Lee L, Klee SK, Evangelista M, Boone C, Pellman D. Control of mitotic spindle position by the Saccharomyces Cerevisiae forming Bnilp. J Cell Biol 1999; 144:947-61.

29 Miller RK, Matheos D, Rose MD. The cortical localization of the microtubule orientation protein Kar9p is dependent upon actin and proteins required for polarization. J Cell Biol 1999; 144:96375.

30 Sardet C, Speksnijder JE, Terasaki M, Chang P. Polarity of ascidian egg cortex before fertilization. Development 1992; 115:221-37.

31 Evans JP, Foster JA, McAvey BA, Gerton GL, Kopf GS, Schultz RM. Effects of perturbation of cell polarity on molecular markers of sperm-egg binding sites on mouse eggs. Biol Reprod 2000; 62:76-84.

32 Longo FJ, Chen DY. Development of surface polarity in mouse eggs. Scan Electron Microsc 1984; (Pt 2):703-16.

33 Longo FJ, Chen DY. Development of cortical polarity in mouse eggs: involvement of the meiotic apparatus. Dev Biol 1985; 107: 382-94.

34 Wassarman PM, Ukena TE, Josefowicz WJ, Karnovsky MJ. Asymmetrical distribution of microvilli in cytochalasin B- induced pseudocleavage of mouse oocytes. Nature 1977; 265:742-4

35 Maro B, Johnson MH, Webb M, Flach G. Mechanism of polar body formation in the mouse oocyte: an interaction between the chromosomes, the cytoskeleton and the plasma membrane. J Embryol Exp Morphol 1986; 92:11-32.

36 Verlhac MH, Lefebvre C, Z.Kubiak J, et al. Mos activates MAP kinase in mouse oocytes through two opposite pathways. EMBO J 2000; 19:6065-74.

37 Gavin AC, Cavadore JC, Schorderet-Slatykine S. Histone H1 kinase activity, germinal vesicle breakdown and $\mathrm{M}$ phase entry in mouse oocytes. J Cell Sci 1994; 107:275-83.

38 Sun QY, Wu GM, Lai LX, et al. Regulation of mitogen-activated protein kinase phosphorylation, microtubule organi-zation, chromatin behavior, and cell cycle progression by protein phosphatases during pig oocyte maturation and fertilization in vitro. Biol Reprod 2002; 66:580-8.

39 Schwartz DA, Schultz RM. Stimulatory effect of okadaic acid, an inhibitor of protein phosphatases, on nuclear envelope breakdown and protein phosphorylation in mouse oocytes and onecell embryos. Dev Biol 1991; 145:119-27.

40 Sohaskey ML, Ferrell JL. Distinct, constitutively active MAPK phosphatase function in Xenopus oocytes: implications for p42 MAPK regulation in vivo. Mol Biol Cell 1999; 10:3729-43.

41 Sun ZG, Kong WH, Zhang YJ, Yan S, Lu JN, Gu Z, Lin F, Tso JK. A novel ubiquitin carboxyl terminal hydrolase is involved in toad oocyte maturation. Cell Res 2002; 12:199-206.

42 Gross SD, Schwab MS, Taieb FE, Lewellyn AL, Qian YW, Maller JL. The critical role of MAP kinase pathway in meiosis II in Xenopus oocytes is mediated by p90rsk. Curr Biol 2000; 10:430-8.

43 Kalab P, Kubiak JZ, Verlhac MH, Colledge WH, Maro B. Activation of p90rsk during meiotic maturation and first mitosis in mouse oocytes and eggs: MAP kinase-independent and -dependent activation. Development 1996; 122:1957-64.

44 Tan X, Chen DY, Yang Z, et al. Phosphorylation of p90rsk during meiotic maturation and parthenogenetic activation of rat oocytes: correlation with MAP kinases. Zygote 2001; 9:269-76.

45 Zhang W, Liu HT. MAPK signal pathways in the regulation of cell proliferation in mammalian cells. Cell Res 2002; 12:9-18. 
Cell Research (2003); 13(5):385-391

http://www.cell-research.com

\title{
Intermittent hypoxia attenuates ischemia/reperfusion induced apoptosis in cardiac myocytes via regulating Bcl-2/Bax expression
}

\author{
Jian Wen Dong1, Hai Feng Zhu1, Wei Zhong Zhu1, Hai Lei Ding1, Tie Min Ma2, Zhao Nian Zhou1* \\ 1 Laboratory of Hypoxic Cardiovascular Physiology, Shanghai Institutes for Biological Sciences, Chinese Academy of \\ Sciences, Shanghai 200031, China.E-mail:znzhou@server.shcnc.ac.cn \\ 2 Department of Physiology and Pathophysiology, Health Science Center Peking University, Beijing 100083, China.
}

\begin{abstract}
Intermittent hypoxia has been shown to provide myocardial protection against ishemia/reperfusion-induced injury. Cardiac myocyte loss through apoptosis has been reported in ischemia/reperfusion injury. Our aim was to investigate whether intermittent hypoxia could attenuate ischemia/reperfusion-induced apoptosis in cardiac myocytes and its potential mechanisms. Adult male Sprague-Dawley rats were exposed to hypoxia simulated $5000 \mathrm{~m}$ in a hypobaric chamber for $6 \mathrm{~h}$ /day, lasting 42 days. Normoxia group rats were kept under normoxic conditions. Isolated perfused hearts from both groups were subjected to $30 \mathrm{~min}$ of global ischemia followed by $60 \mathrm{~min}$ reperfusion. Incidence of apoptosis in cardiac myocytes was determined by terminal deoxynucleotidyl transferase mediated dUTP nick end labeling (TUNEL) and DNA agarose gel electrophoresis. Expressions of apoptosis related proteins, Bax and Bcl-2, in cytosolic and membrane fraction were detected by Western Blotting. After ischemia/reperfusion, enhanced recovery of cardiac function was observed in intermittent hypoxia hearts compared with normoxia group. Ischemia/ reperfusion-induced apoptosis, as evidenced by TUNEL-positive nuclei and DNA fragmentation, was significantly reduced in intermittent hypoxia group compared with normoxia group. After ischemia/reperfusion, expression of Bax in both cytosolic and membrane fractions was decreased in intermittent hypoxia hearts compared with normoxia group. Although ischemia/reperfusion did not induce changes in the level of Bcl-2 expression in cytosolic fraction between intermittent hypoxia and normoxia groups, the expression of Bcl-2 in membrane fraction was upregulated in intermittent hypoxia group compared with normoxia group. These results indicated that the cardioprotection of intermittent hypoxia against ischemia/reperfusion injury appears to be in part due to reduce myocardial apoptosis. Intermittent hypoxia attenuated ischemia/reperfusion-induced apoptosis via increasing the ratio of Bcl-2/Bax, especially in membrane fraction.
\end{abstract}

Keywords: intermittent hypoxia, apoptosis, cardiac myocytes, Bax, Bcl-2.

\section{INTRODUCTION}

Intermittent hypoxia, or periodic exposure to hypoxia interrupted by return to normoxia or less hypoxic conditions, occurs in many circumstances[1]. Many studies showed that intermittent hypoxia might have the cardioprotective effects similar to those observed in ischemic preconditioning[2-8]. A number of studies have

*Correspondence: Prof. Zhao Nian ZHOU, Laboratory of Hypoxic Cardiovascular Physiology, Shanghai Institutes for Biological Sciences, Chinese Academy of Sciences, 320 Yueyang Road, Shanghai 200031, China.

Tel: 86-21-5492-0305, Fax: 86-21-5492-0306

E-mail: znzhou@server.shcnc.ac.cn

Received March-4-2003 Revised Aug-11-2003 Accepted Sep-15-2003 attempted to define the mechanisms of this phenomenon and several potential factors have been proposed to be involved in the protective mechanism afforded by intermittent hypoxia[3,5-7,9-11], however, the precise mechanisms underlying the protective effects of intermittent hypoxia on ischemic hearts are far from clear.

Apoptosis, a form of death characterized by cell shrinkage, plasma membrane blebbing, chromatin condensation and genomic DNA fragmentation, is essential for development and maintenance of tissue homeostasis. On the other hand, apoptosis has been implicated in many diseases such as congestive heart failure and ischemic injury. Although a large portion of the cell loss during cardiac ischemia and reperfusion occurs through 